\title{
BSDES ON FINITE AND INFINITE TIME HORIZON WITH DISCONTINUOUS COEFFICIENTS
}

\author{
Penguu Duan and Yong Ren
}

\begin{abstract}
This paper is devoted to solving one dimensional backward stochastic differential equations (BSDEs). We prove the existence of the solutions to BSDEs if the generator satisfies the general growth and discontinuous conditions.
\end{abstract}

\section{Introduction}

The backward stochastic differential equations (BSDEs for short), in the nonlinear case, were firstly introduced by Pardoux and Peng [10], who established the existence and uniqueness of solutions of BSDEs when the generator is under Lipschitz conditions. Since then, BSDEs have been studied because of the universal applications in stochastic games, partial differential equations and mathematical financial, etc. Owe to the restriction of Lipschitz conditions, many authors improved the results of Pardoux and Peng (see $[2,4,6,8,9]$ ). Particularly, Gia [7] obtained the existence of solutions of one dimensional BSDEs with linear growth and discontinuous assumptions.

Chen and Wang [1] discussed a class of one dimensional BSDEs with infinite time interval and obtained the unique result with suitable conditions. Furthermore, Fan and Jiang [3] studied the existence and uniqueness of solutions of multidimensional BSDEs with non-Lipschitz coefficients, which generalized the result of Chen and Wang [1]. Recently, Fan et al. [5] firstly obtained the existence of minimal solution of one dimensional BSDEs on finite and infinite time horizon with continuous conditions and general growth. Furthermore, they also gave the unique result under non-Lipschitz assumptions.

Motivated by these works, especially by [7] and [5], we are devoted to solving the one dimensional BSDEs on finite and infinite time horizon with discontinuous conditions and general growth of generator. In Section 2, we give some

Received February 10, 2012; Revised December 1, 2012

2010 Mathematics Subject Classification. primary 60H10, 60H30, 60H99.

Key words and phrases. backward stochastic differential equations, existence and uniqueness, comparison theorem, discontinuous conditions.

Sponsored by the NSF of China (10901003); the NSF of Anhui Province of China (KJ2011B176); Foundation of Laboratory of Intelligent Information Processing of Suzhou University (2010YKF11). 
notations and technical lemmas. In Section 3, we put forward and prove our main result.

\section{Notations and lemmas}

Let $0 \leq T \leq+\infty$ be a fixed constant. Let $(\Omega, \mathcal{F}, P)$ be a probability space carrying a standard $d$-dimensional Brownian motion $\left(W_{t}\right)_{t \geq 0},\left(\mathcal{F}_{t}\right)_{t \geq 0}$ be the natural $\sigma$-algebra generated by $\left(W_{t}\right)_{t \geq 0}$ and assume $\mathcal{F}_{T}=\overline{\mathcal{F}}$. $|\cdot|$ denotes the Euclidean norm in $\mathbf{R}^{n}$. Next, we propose some spaces as follows:

$L^{2}\left(\Omega, \mathcal{F}_{t}, P\right)$ denotes the set of all $\mathcal{F}_{t}$-measurable random variable $\xi$ such that $E|\xi|^{2}<\infty$.

$\mathcal{L}^{2}(0, T ; \mathbf{R})$ denotes the set of $\mathcal{F}_{t}$-progressively measurable $\mathbf{R}$-valued process $\left\{\varphi_{t}, t \in[0, T]\right\}$ such that

$$
\|\varphi\|^{2}:=\mathbf{E}\left[\left(\int_{0}^{T} \varphi_{t} \mathrm{dt}\right)^{2}\right]<\infty .
$$

$\mathbf{M}^{2}\left(0, T ; \mathbf{R}^{n}\right)$ denotes the set of $\mathcal{F}_{t}$-progressively measurable $\mathbf{R}^{n}$-valued process $\left\{z_{t}, t \in[0, T]\right\}$ such that

$$
\|z\|_{M^{2}}^{2}:=\mathbf{E}\left[\int_{0}^{T}\left|z_{t}\right|^{2} \mathrm{dt}\right]<\infty
$$

$S^{2}(0, T ; \mathbf{R})$ denotes the set of real valued, adapted and continuous process $\left(y_{t}\right)_{t \in[0, T]}$ such that

$$
\left\|y_{t}\right\|_{S^{2}}^{2}:=\mathbf{E}\left[\sup _{t \in[0, T]}\left|y_{t}\right|^{2}\right]<\infty .
$$

In this paper, we mainly discuss the following one dimensional backward stochastic differential equations

$$
y_{t}=\xi+\int_{t}^{T} g\left(s, y_{s}, z_{s}\right) \mathrm{d} s-\int_{t}^{T} z_{s} \mathrm{~d} W_{s}, t \in[0, T]
$$

where $\xi \in L^{2}(\Omega, \mathcal{F}, P)$, the generator $g(\omega, t, y, z): \Omega \times[0, T] \times \mathbf{R} \times \mathbf{R}^{d} \rightarrow \mathbf{R}$ is progressively measurable for each $(y, z)$.

Definition 2.1. A pair of process $\left(y_{t}, z_{t}\right)_{t \in[0, T]}$ is called a solution to BSDE $(2.1)$, if $\left(y_{t}, z_{t}\right)_{t \in[0, T]} \in S^{2}(0, T ; \mathbf{R}) \times M^{2}\left(0, T ; \mathbf{R}^{d}\right)$ which satisfies BSDE (2.1).

In the following, we introduce some assumptions with respect to the generator of one dimensional BSDE (2.1) with $0 \leq T \leq+\infty$ :

(H1) $g(t, \cdot, z)$ is left continuous and $g(t, y, \cdot)$ is continuous;

(H2) There exist two deterministic functions $u(\cdot), v(\cdot):[0, T] \rightarrow \mathbf{R}^{+}$with $\int_{0}^{T}\left[u(t)+v^{2}(t)\right] \mathrm{d} t<+\infty$ and an $\mathcal{F}_{t}$-progressively measurable, nonnegative process $\left\{f_{t}\right\}_{t \in[0, T]}$ with $\mathbf{E}\left[\left(\int_{0}^{T} f_{t} \mathrm{~d} t\right)^{2}\right]<+\infty$ such that for all $y \in \mathbf{R}, z \in \mathbf{R}^{d}$,

$$
|g(t, y, z)| \leq f_{t}+u(t)|y|+v(t)|z|, \mathrm{d} P \times \mathrm{d} t \text {-a.s. }
$$


(H3) There exists a continuous function $\kappa(\cdot, \cdot): \mathbf{R} \times \mathbf{R}^{d} \rightarrow \mathbf{R}$ which satisfies $|\kappa(y, z)| \leq u(t)|y|+v(t)|z|$ for all $y_{1} \geq y_{2} \in \mathbf{R}, z_{1}, z_{2} \in \mathbf{R}^{d}$, such that

$$
g\left(t, y_{1}, z_{1}\right)-g\left(t, y_{2}, z_{2}\right) \geq \kappa\left(y_{1}-y_{2}, z_{1}-z_{2}\right) .
$$

For the sake of convenience, we introduce some technical lemmas. The following Lemma 2.2 appears in Fan et al. [5].

Lemma 2.2. Assume that the generator $g(t, y, z)$ of $B S D E(2.1)$ is continuous in $(y, z)$ and $(\mathrm{H} 2)$ holds, then, for each $\xi \in L^{2}\left(\Omega, \mathcal{F}_{T}, P\right)$, the BSDE (2.1) has a minimal solution $\left(y_{t}, z_{t}\right)_{t \in[0, T]}$.

Lemma 2.3. Assume (H3) holds, let $\kappa_{n}(y, z)$ be defined as

$$
\kappa_{n}(y, z)=\inf _{(u, v) \in \boldsymbol{R}^{1+d}}\{\kappa(y, z)+n u(t)|y-u|+n v(t)|z-v|\} .
$$

Then, the sequence of functions $\kappa_{n}(y, z)$ is well defined for each $n \geq 1$, and it satisfies

(i) General growth: $\left|\kappa_{n}(y, z)\right| \leq u(t)|y|+v(t)|z|$;

(ii) Monotonicity: $\kappa_{n}(y, z)$ increases in $n$;

(iii) Lipschitz condition:

$$
\left|\kappa_{n}\left(y_{1}, z_{1}\right)-\kappa_{n}\left(y_{2}, z_{2}\right)\right| \leq n u(t)\left|y_{1}-y_{2}\right|+n v(t)\left|z_{1}-z_{2}\right|
$$

(iv) Convergence: If $\left(y_{n}, z_{n}\right) \rightarrow(y, z), \kappa_{n}\left(y_{n}, z_{n}\right) \rightarrow \kappa(y, z)$.

The proof is similar to Lemma 1 in [8], we omit it.

Lemma 2.4. Let $\kappa(y, z)$ be defined in (H3), we consider the following BSDE

$$
y_{t}=\xi+\int_{t}^{T}\left[\kappa\left(y_{s}, z_{s}\right)+\phi(s)\right] \mathrm{d} s-\int_{t}^{T} z_{s} \mathrm{~d} W_{s}, t \in[0, T],
$$

where $\xi \in L^{2}\left(\Omega, \mathcal{F}_{T}, P\right)$, and $\phi(s) \in \mathcal{L}^{2}(0, T ; \boldsymbol{R})$. Then,

(i) Eq.(2.3) admits a minimal solution $\left(\underline{y}_{t}, \underline{z}_{t}\right) \in S^{2}(0, T ; \boldsymbol{R}) \times M^{2}\left(0, T ; \boldsymbol{R}^{d}\right)$;

(ii) For any solution of $B S D E(2.3), \phi \bar{t} t) \geq 0$ and $\xi \geq 0$, then it implies $\underline{y}_{t} \geq 0, P$-a.s.

Proof. From Lemma 2.2, we can derive that BSDE (2.3) has at least one solution. In order to complete the proof, we consider the following three equations

$$
\begin{aligned}
& y_{t}^{1}=\xi+\int_{t}^{T}\left[-u(s)\left|y_{s}^{1}\right|-v(s)\left|z_{s}^{1}\right|\right] \mathrm{d} s-\int_{t}^{T} z_{s}^{1} \mathrm{~d} W_{s}, t \in[0, T], \\
& y_{t}^{2}=\int_{t}^{T}\left[-u(s)\left|y_{s}^{2}\right|-v(s)\left|z_{s}^{2}\right|\right] \mathrm{d} s-\int_{t}^{T} z_{s}^{2} \mathrm{~d} W_{s}, t \in[0, T],
\end{aligned}
$$

and

$$
y_{t}^{n}=\xi+\int_{t}^{T}\left[\kappa_{n}\left(y_{s}^{n}, z_{s}^{n}\right)+\phi(s)\right] \mathrm{d} s-\int_{t}^{T} z_{s}^{n} \mathrm{~d} W_{s}, t \in[0, T], n \geq 3 .
$$


By Theorem 1.2 of Chen and Wang [1], we know each of the above three equations has a unique solution. Let $\left(y_{t}^{1}, z_{t}^{1}\right),\left(y_{t}^{2}, z_{t}^{2}\right)$ and $\left(y_{t}^{n}, z_{t}^{n}\right)$ be solution of (2.4), (2.5) and (2.6), respectively. By the comparison theorem (see Fan et al. [5]), it implies

$$
y_{t}^{n} \geq y_{t}^{1} \geq y_{t}^{2}, t \in[0, T] .
$$

Because (2.5) has a unique solution, $(0,0)$ is a solution, $y_{t}^{2}=0$. Moreover, from Lemma 2.2, the solution $\left\{\left(y_{t}^{n}, z_{t}^{n}\right)\right\}_{n=1}^{\infty}$ of (2.6) converges to the minimal solution $\left(\underline{y}_{t}, \underline{z}_{t}\right)$. Thus, we have

$$
\underline{y}_{t} \geq y_{t}^{1} \geq y_{t}^{2}=0, t \in[0, T] .
$$

So the proof is completed.

In order to get the main result of this paper, we construct a sequence of BSDEs as follows:

$$
\begin{aligned}
\underline{y}_{t}^{0}=\xi & +\int_{t}^{T}\left[-u(s)\left|\underline{y}_{s}^{0}\right|-v(s)\left|\underline{z}_{s}^{0}\right|-f_{s}\right] \mathrm{d} s-\int_{t}^{T} \underline{z}_{s}^{0} \mathrm{~d} W_{s}, t \in[0, T], \\
\underline{y}_{t}^{i}=\xi & +\int_{t}^{T}\left[g\left(s, \underline{y}_{s}^{i-1}, \underline{z}_{s}^{i-1}\right)+\kappa\left(\underline{y}_{s}^{i}-\underline{y}_{s}^{i-1}, \underline{z}_{s}^{i}-\underline{z}_{s}^{i-1}\right)\right] \mathrm{d} s \\
& -\int_{t}^{T} \underline{z}_{s}^{i} \mathrm{~d} W_{s}, t \in[0, T], i=1,2, \ldots,
\end{aligned}
$$

and

$$
\bar{y}_{t}^{0}=\xi+\int_{t}^{T}\left[u(s)\left|\bar{y}_{s}^{0}\right|+v(s)\left|\bar{z}_{s}^{0}\right|+f_{s}\right] \mathrm{d} s-\int_{t}^{T} \bar{z}_{s}^{0} \mathrm{~d} W_{s}, t \in[0, T] .
$$

By the existence Theorem 1.2 in Chen and Wang [1], both the equations (2.8) and (2.10) have unique solutions which are denoted by $\left(\underline{y}_{t}^{0}, \underline{z}_{t}^{0}\right)$ and $\left(\bar{y}_{t}^{0}, \bar{z}_{t}^{0}\right)$, respectively. In addition, Lemma 2.4 guarantees that (2.9) admits a solution. In the following, we only consider the minimal solution of (2.9), which is denoted by $\left(\underline{y}_{t}^{i}, \underline{z}_{t}^{i}\right)$.

Lemma 2.5. Under the conditions of (H1)-(H3), the solutions of (2.8), (2.9) and (2.10) have the properties, for any $i \in \boldsymbol{N}^{+}, t \in[0, T]$,

$$
\underline{y}_{t}^{0} \leq \underline{y}_{t}^{i} \leq \underline{y}_{t}^{i+1} \leq \bar{y}_{t}^{0}, \text { P-a.s. }
$$

Proof. From (2.8) and (2.9), we have

$$
\underline{y}_{t}^{1}-\underline{y}_{t}^{0}=\int_{t}^{T}\left[\kappa\left(\underline{y}_{s}^{1}-\underline{y}_{s}^{0}, \underline{z}_{s}^{1}-\underline{z}_{s}^{0}\right)+\Delta_{s}^{1}\right] \mathrm{d} s-\int_{t}^{T}\left(\underline{z}_{s}^{1}-\underline{z}_{s}^{0}\right) \mathrm{d} W_{s},
$$

where $\Delta_{s}^{1}=g\left(s, \underline{y}_{s}^{0}, \underline{z}_{s}^{0}\right)+u(s)\left|\underline{y}_{s}^{0}\right|+v(s)\left|\underline{z}_{s}^{0}\right|+f_{s}$. From (H2) and (H3), it implies $\Delta_{s}^{1} \geq 0$ and $\Delta_{s}^{1} \in \mathcal{L}^{2}(0, T ; \mathbf{R})$. According to Lemma 2.4 , we know $\underline{y}_{t}^{0} \leq \underline{y}_{t}^{1}$. 
Next, we assume $\underline{y}_{t}^{i-1} \leq \underline{y}_{t}^{i}$. By (2.9), we can deduce

$$
\underline{y}_{t}^{i+1}-\underline{y}_{t}^{i}=\int_{t}^{T}\left[\kappa\left(\underline{y}_{s}^{i+1}-\underline{y}_{s}^{i}, \underline{z}_{s}^{i+1}-\underline{z}_{s}^{i}\right)+\Delta_{s}^{i+1}\right] \mathrm{d} s-\int_{t}^{T}\left(\underline{z}_{s}^{i+1}-\underline{z}_{s}^{i}\right) \mathrm{d} W_{s},
$$

where $\Delta_{s}^{i+1}=g\left(s, \underline{y}_{s}^{i}, \underline{z}_{s}^{i}\right)-g\left(s, \underline{y}_{s}^{i-1}, \underline{z}_{s}^{i-1}\right)-\kappa\left(\underline{y}_{s}^{i}-\underline{y}_{s}^{i-1}, \underline{z}_{s}^{i}-\underline{z}_{s}^{i-1}\right)$. From (H2) and (H3), it implies $\Delta_{s}^{i+1} \geq 0$ and $\Delta_{s}^{i+1} \in \overline{\mathcal{L}}^{2}(0, T ; \mathbf{R})$, so $\underline{y}_{t}^{i} \leq \underline{y}_{t}^{i+1}$. By mathematical induction, we have

$$
\underline{y}_{t}^{0} \leq \underline{y}_{t}^{i} \leq \underline{y}_{t}^{i+1}, P \text {-a.s. }
$$

We use mathematical induction again to prove $\underline{y}_{t}^{i} \leq \bar{y}_{t}^{0}, i=0,1,2, \ldots$ From (2.8) and (2.10), we have

$$
\begin{aligned}
\bar{y}_{t}^{0}-\underline{y}_{t}^{0}= & \int_{t}^{T}\left\{u(s)\left[\left|\bar{y}_{s}^{0}\right|+\left|\underline{y}_{s}^{0}\right|\right]+v(s)\left[\left|\bar{z}_{s}^{0}\right|+\left|\underline{z}_{s}^{0}\right|\right]+2 f_{s}\right\} \mathrm{d} s \\
& -\int_{t}^{T}\left(\bar{z}_{s}^{0}-\underline{z}_{s}^{0}\right) \mathrm{d} W_{s} .
\end{aligned}
$$

Since $u(s)\left[\left|\bar{y}_{s}^{0}\right|+\left|\underline{y}_{s}^{0}\right|\right]+v(s)\left[\left|\bar{z}_{s}^{0}\right|+\left|\underline{z}_{s}^{0}\right|\right]+2 f_{s} \geq 0$, from Lemma 2.4, we get

$$
\bar{y}_{t}^{0} \geq \underline{y}_{t}^{0}, P \text {-a.s. }
$$

According to (2.9) and (2.10), we have

$$
\begin{aligned}
& \bar{y}_{t}^{0}-\underline{y}_{t}^{1} \\
= & \int_{t}^{T}\left\{u(s)\left|\bar{y}_{s}^{0}\right|+v(s)\left|\bar{z}_{s}^{0}\right|+f_{s}-g\left(s, \underline{y}_{s}^{0}, \underline{z}_{s}^{0}\right)-\kappa\left(\underline{y}_{s}^{1}-\underline{y}_{s}^{0}, \underline{z}_{s}^{1}-\underline{z}_{s}^{0}\right)\right\} \mathrm{d} s \\
& -\int_{t}^{T}\left(\bar{z}_{s}^{0}-\underline{z}_{s}^{1}\right) \mathrm{d} W_{s} \\
= & \int_{t}^{T}\left\{-u(s)\left|\bar{y}_{s}^{0}-\underline{y}_{s}^{1}\right|-v(s)\left|\bar{z}_{s}^{0}-\underline{z}_{s}^{1}\right|+\Psi_{s}^{1}\right\} \mathrm{d} s-\int_{t}^{T}\left(\bar{z}_{s}^{0}-\underline{z}_{s}^{1}\right) \mathrm{d} W_{s},
\end{aligned}
$$

where $\Psi_{s}^{1}=u(s)\left|\bar{y}_{s}^{0}-\underline{y}_{s}^{1}\right|+v(s)\left|\bar{z}_{s}^{0}-\underline{z}_{s}^{1}\right|+u(s)\left|\bar{y}_{s}^{0}\right|+v(s)\left|\bar{z}_{s}^{0}\right|+f_{s}-g\left(s, \underline{y}_{s}^{0}, \underline{z}_{s}^{0}\right)-$ $\kappa\left(\underline{y}_{s}^{1}-\underline{y}_{s}^{0}, \underline{z}_{s}^{1}-\underline{z}_{s}^{0}\right)$. From (H2) and (H3), $\Psi_{s}^{1} \geq g\left(s, \underline{y}_{s}^{1}, \underline{z}_{s}^{1}\right)-g\left(s, \underline{y}_{s}^{0}, \underline{z}_{s}^{0}\right)-\kappa\left(\underline{y}_{s}^{1}-\right.$ $\left.\underline{y}_{s}^{0}, \underline{z}_{s}^{1}-\underline{z}_{s}^{0}\right) \geq 0$. By Lemma 2.4 , we have $\underline{y}_{t}^{1} \leq \bar{y}_{t}^{0}, P$-a.s.

Assume $\underline{y}_{t}^{i} \leq \bar{y}_{t}^{0}$, by (2.9) and (2.10), we have

$$
\begin{aligned}
& \bar{y}_{t}^{0}-\underline{y}_{t}^{i+1} \\
= & \int_{t}^{T}\left\{-u(s)\left|\bar{y}_{s}^{0}-\underline{y}_{s}^{i+1}\right|-v(s)\left|\bar{z}_{s}^{0}-\underline{z}_{s}^{i+1}\right|+\Psi_{s}^{i+1}\right\} \mathrm{d} s-\int_{t}^{T}\left(\bar{z}_{s}^{0}-\underline{z}_{s}^{i+1}\right) \mathrm{d} W_{s},
\end{aligned}
$$

where $\Psi_{s}^{i+1}=u(s)\left|\bar{y}_{s}^{0}-\underline{y}_{s}^{i+1}\right|+v(s)\left|\bar{z}_{s}^{0}-\underline{z}_{s}^{i+1}\right|+u(s)\left|\bar{y}_{s}^{0}\right|+v(s)\left|\bar{z}_{s}^{0}\right|+f_{s}-$ $g\left(s, \underline{y}_{s}^{i}, \underline{z}_{s}^{i}\right)-\kappa\left(\underline{y}_{s}^{i+1}-\underline{y}_{s}^{i}, \underline{z}_{s}^{i+1}-\underline{z}_{s}^{i}\right)$. Similar proof as above, it follows $\underline{y}_{t}^{i+1} \leq$ $\bar{y}_{t}^{0}, \bar{P}_{\text {-a.s. }}$

So, the proof is complete. 


\section{Main result}

Now, we give our main result.

Theorem 3.1. Under assumptions (H1)-(H3), the solutions $\left(\underline{y}_{s}^{i}, \underline{z}_{t}^{i}\right)_{i=1}^{\infty}$ of $(2.9)$ converge to $\left(\underline{y}_{t}, \underline{z}_{t}\right)$, which is a solution of $(2.1)$.

Proof. From Lemma 2.5, we know $\left\{\underline{y}_{s}^{n}\right\}$ is increasing, and bounded in $S^{2}(0, T$; $\mathbf{R})$. By dominated convergence theorem, we can imply $\left\{\underline{y}_{t}^{i}\right\}_{i=1}^{\infty}$ converges in $S^{2}(0, T ; \mathbf{R})$ to a limit $\underline{y}_{t}$, and

$$
\sup _{i} \mathbf{E}\left[\sup _{0 \leq t \leq T}\left|\underline{y}_{s}^{i}\right|^{2}\right] \leq \mathbf{E}\left[\sup _{0 \leq t \leq T}\left|\underline{y}_{t}^{0}\right|^{2}\right]+\mathbf{E}\left[\sup _{0 \leq t \leq T}\left|\bar{y}_{t}^{0}\right|^{2}\right]<\infty .
$$

Applying Itô formula to $\left|\underline{y}_{t}^{i+1}\right|^{2}$, we have

$$
\begin{aligned}
& \mathbf{E}\left[\left|\underline{y}_{T}^{i+1}\right|^{2}\right] \\
= & \mathbf{E}\left[\left|\underline{y}_{0}^{i+1}\right|^{2}\right]+\mathbf{E} \int_{0}^{T}\left\{\left|\underline{z}_{t}^{i+1}\right|^{2}-2 \underline{y}_{t}^{i+1}\left[g\left(t, \underline{y}_{t}^{i}, \underline{z}_{t}^{i}\right)+\kappa\left(\underline{y}_{t}^{i+1}-\underline{y}_{t}^{i}, \underline{z}_{t}^{i+1}-\underline{z}_{t}^{i}\right)\right]\right\} \mathrm{d} t .
\end{aligned}
$$

Let $G(\omega)=\sup _{n} \sup _{s \in[0, T]}\left|y_{s}^{n}(\omega)\right|, \lambda=2 \int_{0}^{T} v^{2}(t) \mathrm{d} t$. From the assumption of (H2) and (H3), we get

$$
\begin{aligned}
& \mathbf{E} \int_{0}^{T}\left|\underline{z}_{t}^{i+1}\right|^{2} \mathrm{~d} t \\
= & \mathbf{E}|\xi|^{2}+2 \mathbf{E} \int_{0}^{T} \underline{y}_{t}^{i+1}\left[g\left(t, \underline{y}_{t}^{i}, \underline{z}_{t}^{i}\right)+\kappa\left(\underline{y}_{t}^{i+1}-\underline{y}_{t}^{i}, \underline{z}_{t}^{i+1}-\underline{z}_{t}^{i}\right)\right] \mathrm{d} t \\
\leq & \mathbf{E}|\xi|^{2}+2 \mathbf{E} \int_{0}^{T}\left|\underline{y}_{t}^{i+1}\right|\left[\left|g\left(t, \underline{y}_{t}^{i}, \underline{z}_{t}^{i}\right)\right|+\left|\kappa\left(\underline{y}_{t}^{i+1}-\underline{y}_{t}^{i}, \underline{z}_{t}^{i+1}-\underline{z}_{t}^{i}\right)\right|\right] \mathrm{d} t \\
\leq & \left.\mathbf{E}|\xi|^{2}+2 \mathbf{E} \int_{0}^{T}\left|\underline{y}_{t}^{i+1}\right|\left[f_{t}+u(t)\left|\underline{y}_{t}^{i}\right|+v(t) \mid \underline{z}_{t}^{i}\right)|+u(t)| \underline{y}_{t}^{i+1}-\underline{y}_{t}^{i}|+v(t)| \underline{z}_{t}^{i+1}-\underline{z}_{t}^{i} \mid\right] \mathrm{d} t .
\end{aligned}
$$

By using the inequalities $2 a b \leq a^{2}+b^{2}, 2 a b \leq \beta a^{2}+\frac{1}{\beta} b^{2}$ for $\beta>0$, we have

$$
\begin{aligned}
\mathbf{E} \int_{0}^{T}\left|\underline{z}_{t}^{i+1}\right|^{2} \mathrm{~d} t \leq & \mathbf{E}|\xi|^{2}+\left(1+24 \lambda+4 \int_{0}^{T} u(t) \mathrm{d} t\right) \mathbf{E} G^{2}+\mathbf{E}\left[\int_{0}^{T} f_{t} \mathrm{~d} t\right]^{2} \\
& +\frac{1}{4} \mathbf{E} \int_{0}^{T}\left[\left|\underline{z}_{t}^{i+1}\right|^{2}+\left|\underline{z}_{t}^{i}\right|^{2}\right] \mathrm{d} t \\
= & C_{1}+\frac{1}{4} \mathbf{E} \int_{0}^{T}\left[\left|\underline{z}_{t}^{i+1}\right|^{2}+\left|\underline{z}_{t}^{i}\right|^{2}\right] \mathrm{d} t
\end{aligned}
$$

Thus, $\sup _{i} \mathbf{E} \int_{0}^{T}\left|\underline{z}_{t}^{i}\right|^{2} \mathrm{~d} t<\infty$, which leads to $\Lambda_{t}^{i+1}:=g\left(s, \underline{y}_{t}^{i}, \underline{z}_{s}^{i}\right)+\kappa\left(\underline{y}_{s}^{i+1}-\right.$ $\left.\underline{y}_{t}^{i}, \underline{z}_{t}^{i+1}-\underline{z}_{t}^{i}\right)$ be uniformly bounded in $\mathcal{L}^{2}(0, T ; \mathbf{R})$. Applying Itô formula to 
$\left|\underline{y}_{t}^{n}-\underline{y}_{t}^{m}\right|^{2}$, as the same proof of Theorem 1 in Fan et al. [5], we have

$$
\begin{aligned}
\mathbf{E}\left|\underline{y}_{t}^{n}-\underline{y}_{t}^{m}\right|^{2}+\mathbf{E} \int_{t}^{T}\left|\underline{z}_{s}^{n}-\underline{z}_{s}^{m}\right|^{2} \mathrm{~d} s & =2 \mathbf{E} \int_{t}^{T}\left(\underline{y}_{s}^{n}-\underline{y}_{s}^{m}\right)\left(\Lambda_{s}^{n}-\Lambda_{s}^{m}\right) \mathrm{d} s \\
& \leq 2 \mathbf{E} \int_{t}^{T}\left|\underline{y}_{s}^{n}-\underline{y}_{s}^{m}\right|\left[\left|\Lambda_{s}^{n}\right|+\left|\Lambda_{s}^{m}\right|\right] \mathrm{d} s .
\end{aligned}
$$

From the conditions (H2) and (H3), we have

$$
\begin{aligned}
& \mathbf{E}\left|\underline{y}_{t}^{n}-\underline{y}_{t}^{m}\right|^{2}+\mathbf{E} \int_{t}^{T}\left|\underline{z}_{s}^{n}-\underline{z}_{s}^{m}\right|^{2} \mathrm{~d} s \\
\leq & 4 \mathbf{E}\left[\int_{t}^{T}\left|\underline{y}_{s}^{n}-\underline{y}_{s}^{m}\right| f_{s} \mathrm{~d} s\right]+8 \sqrt{\mathbf{E}\left[G^{2}\right]} \cdot \sqrt{\mathbf{E}\left[\int_{t}^{T}\left|\underline{y}_{s}^{n}-\underline{y}_{s}^{m}\right| u(s) \mathrm{d} s\right]^{2}} \\
& +8 \sqrt{C_{1}} \sqrt{\mathbf{E}\left[G^{2}\right]} \cdot \sqrt{\mathbf{E}\left[\int_{t}^{T}\left|\underline{y}_{s}^{n}-\underline{y}_{s}^{m}\right|^{2} v^{2}(s) \mathrm{d} s\right]} .
\end{aligned}
$$

Therefore, Lebesgue dominated convergence theorem yields $\left\{\underline{z}_{t}^{i}\right\}_{i=1}^{\infty}$ is a Cauchy sequence and converges in $\mathbf{M}^{2}\left(0, T ; \mathbf{R}^{d}\right)$, we denote it by $\underline{z}_{t}$.

Now, we take limits as $n \rightarrow \infty$ in (2.9), then

$$
\underline{y}_{t}=\xi+\int_{t}^{T} g\left(s, \underline{y}_{s}, \underline{z}_{s}\right) \mathrm{d} s-\int_{t}^{T} \underline{z}_{s} \mathrm{~d} W_{s}, t \in[0, T] .
$$

Thus, $\left\{\underline{y}_{t}, \underline{z}_{t}\right\}_{t \in[0, T]}$ satisfies $(2.1)$.

Remark. If $0 \leq T<+\infty$, let $u(t), v(t)=A$, the result of this paper includes the result of Lepeltier and San Martin [8] and Theorem 3 in [7].

If $(\mathrm{H} 1)$ is replaced by the following condition

$\left(\mathrm{H} 1^{\prime}\right) g(t, \cdot, z)$ is right continuous, and $g(t, y, \cdot)$ is continuous, we can get:

Theorem 3.2. Under the assumptions $\left(\mathrm{H} 1^{\prime}\right)$, (H2) and (H3), then the Eq.(2.1) has a solution in $S^{2}(0, T ; \boldsymbol{R}) \times M^{2}\left(0, T ; \boldsymbol{R}^{d}\right)$.

In order to complete the proof, we consider (2.10) and the following equation

$$
\begin{aligned}
\underline{y}_{t}^{i}=\xi & +\int_{t}^{T}\left[g\left(s, \underline{y}_{s}^{i}, \underline{z}_{s}^{i}\right)-\kappa\left(\underline{y}_{s}^{i}-\underline{y}_{s}^{i-1}, \underline{z}_{s}^{i}-\underline{z}_{s}^{i-1}\right)\right] \mathrm{d} s \\
& -\int_{t}^{T} \underline{z}_{s}^{i} \mathrm{~d} W s, t \in[0, T], i=1,2, \ldots
\end{aligned}
$$

By similar procedures in Lemma 2.4, Lemma 2.5 and Theorem 3.1, we can get it.

Acknowledgement. The author would like to thank his/her referee for the valuable comments. 


\section{References}

[1] Z. Chen and B. Wang, Infinite time interval BSDEs and the convergence of $g$ martingales, J. Austral. Math. Soc. Ser. A 69 (2000), no. 2, 187-211.

2] S. Fan and L. Jiang, Uniqueness result for the BSDE whose generator is monotonic in $y$ and uniformly continuous in z, C. R. Math. Acad. Sci. Paris 348 (2010), no. 1-2, 89-92.

[3] - Finite and infinite time interval BSDEs with non-Lipschitz coefficients, Statist. Probab. Lett. 80 (2010), no. 11-12, 962-968.

[4] S. Fan, L. Jiang, and M. Davison, Uniqueness of solutions for multidimensional BSDEs with uniformly continuous generators, C. R. Math. Acad. Sci. Paris 348 (2010), no. 11-12, 683-686.

[5] S. Fan, L. Jiang, and D. Tian, One-dimensional BSDEs with finite and infinite time horizons, Stochastic Process. Appl. 121 (2011), no. 3, 427-440.

[6] S. Hamadène, Multidimensional backward stochastic differential equations with uniformly continuous coefficient, Bernoulli 9 (2003), no. 3, 517-534.

[7] G. Jia, A class of backward stochastic differential equations with discontinuous coefficients, Statist. Probab. Lett. 78 (2008), no. 3, 231-237.

[8] J. P. Lepeltier and J. San Martin, Backward stochastic differential with continuous coefficients, Statist. Probab. Lett. 32 (1997), no. 4, 426-430.

[9] X. Mao, Adapted solutions of backward stochastic differential equations with nonLipschitz coefficients, Stochastic Process. Appl. 58 (1995), no. 2, 281-292.

[10] E. Pardoux and S. Peng, Adapted solution of a backward stochastic differential equation, Systems Control. Lett. 14 (1990), no. 1, 55-61.

PenguU DuAN

Laboratory of Intelligent Information Processing

SuZHOU UNIVERSITY

Anhui, 234000, P. R. China

AND

SChool of Mathematics and Statistics

SuZHOU UNIVERSITY

Anhui 234000, P. R. ChinA

E-mail address: pjduan1981@hotmail.com

YONG REN

Department of Mathematics

Anhui Normal University

Wuhu Anhui 241000, P. R. China

E-mail address: brightry@hotmail.com 